\title{
Doctor, My Knee Hurts!
}

\author{
Yasser Alwabli* \\ Assistant Professor of Orthopaedic Surgery, Qassim University, Saudi Arabia \\ *Corresponding Author: Yasser Alwabli, Assistant Professor of Orthopaedic Surgery, Qassim University, Saudi Arabia.
}

Received: September 25, 2019; Published: November 01, 2019

DOI: 10.31080/ASOR.2019.02.0121

Knee pain is one of the most frequent musculoskeletal issues patients usually present to health care facilities seeking medical advice. Patients with this kind of complaints typically present to different clinical settings such as primary care centres, family physicians, or orthopaedic clinics. The underlying causes of knee pain are diverse, and a thorough knowledge of the anatomy and pathophysiology of the knee will help clinicians at reaching the correct diagnosis and treatment. The importance of understanding knee pathologies and its different treatments should be stressed at all levels of medical practice; beginning from undergraduate education and progressing towards specialty postgraduate training, and continuing medical education.

Clinicians should be equipped with the needed skills and knowledge to manage patients with knee pains. Additionally, the standard medical practices of history taking, physical examination, and investigations should all be carried out on each clinical setting to ensure the accuracy of the diagnosis and proper treatment choices. As a result, and by following these medical standards, patients with knee pains are more likely to receive a correct diagnosis and proper treatment plan. Furthermore, medical institutions need to follow internationally accepted standards of care for the diagnosis and treatment of different knee pathologies. If this is deemed unattainable, then local or national standards of care should be established and adopted by national medical centres. All health care centres should be held accountable for following national medical standards.

Clinicians and future doctors have to understand that knee pain in a young patient is entirely different from a similar pain in an older patient. Whenever a young patient comes in with knee pain, pathologies like trauma or OCD (osteochondritis dissecans), for example, should be ruled out first. Conversely, an older patient with knee pain has an utterly different etiologist that clinicians need to investigate and treat. Degenerative arthritic changes are extremely common in this age group; however, other, more serious conditions, need to be also investigated and treated, such as malignancies or other serious diseases. Similarly, less common conditions should always be kept in mind in the evaluation of an older patient with knee pain.

Due to the vast array of the underlying causes of knee pain, treatment is often individualized to each patient needs. Also, due to the prevalent nature of this complaint, there are various treatment options available for clinicians to manage different underlying pathologies of this complaint. As with any other medical conditions, non-surgical and surgical treatment options are available for clinicians in treating the contributing pathology. With the advancement of medicine and surgery, there is now a designated sub-specialty for orthopaedic surgeons to specialize in knee surgery to perform different surgeries aiming at treating different knee pathologies that cause knee pain.

Training for future doctors on the management of this common issue is fundamental in medical education. Organizers and planners of medical education curricula are required to embed this topic in the undergraduate and postgraduate medical education programs. Of particular emphasis, postgraduate trainees in family medicine and orthopaedic surgery need specific training on how to manage this condition either surgically or non-surgically. Training on advanced surgical skills is also needed as more old patients are diagnosed with knee arthritis, and more young patients are diagnosed with sport-related knee injuries.

\section{Volume 2 Issue 12 December 2019 (C) All rights are reserved by Yasser Alwabli.}

